Check for updates

Cite this: RSC Adv., 2017, 7, 38884

Received 8th May 2017

Accepted 28th July 2017

DOI: $10.1039 / \mathrm{c} 7 \mathrm{ra0} 5167 \mathrm{k}$

rsc.li/rsc-advances

\section{A facile molecularly imprinted electrochemical sensor based on graphene: application to the selective determination of thiamethoxam in grain $\uparrow$}

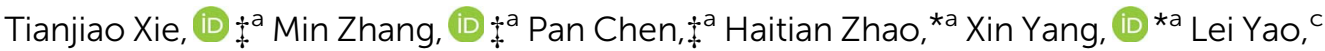 \\ Hua Zhang, ${ }^{a}$ Aijun Dong, ${ }^{a}$ Jing Wang ${ }^{* b}$ and Zhenyu Wang ${ }^{a}$
}

In this study, we report a facile method for the preparation of molecularly imprinted polymer based graphene for the electrochemical detection of thiamethoxam residue. We choose $p$-vinylbenzoic acid as a functional monomer; it can be used to add recognition units and alkenyl units onto the surface of graphene via $\pi-\pi$ interactions in just one step, effectively simplifying the reaction process. And an ultrathin imprinting film $(2 \mathrm{~nm})$ is formed by reducing the addition step involving the alkenyl modifier. Due to the irreversible electrochemical reduction characteristics of thiamethoxam, the template can be removed easily using cyclic voltammetry scanning, without the need for organic solvents. The performance of the fabricated sensor was evaluated and the results indicated that the sensor exhibited excellent specific recognition abilities for thiamethoxam detection; the imprinting factor is 2.36 . The peak current from thiamethoxam is linearly proportional to its concentration over the range from 0.5 to $20 \mu \mathrm{M}$, and the detection limit is $0.04 \mu \mathrm{M}$. The practical application of the sensor was also realized in the selective detection of thiamethoxam in real samples.

\section{Introduction}

Thiamethoxam (TMX) is a representative compound of new neonicotinoid pesticides, which are the main pesticides used to control rice pests. Thiamethoxam has the characteristics of having high efficiency, low toxicity, a wide insecticide spectrum and long persistence. It is widely used in the world. ${ }^{1-4}$ However, the impact of residues of thiamethoxam on the environment, and its effect on blindness, human health, and non-target organisms have caused widespread worldwide attention ${ }^{5}$. At present, many countries have established maximum residue limits (MRLs) for thiamethoxam in food. In Japan, the MRL for thiamethoxam in brown rice is $0.1 \mathrm{mg} \mathrm{kg}{ }^{-1}$. The national standard for food safety published in China stipulates that the MRLs for thiamethoxam in cucumber and brown rice are $0.5 \mathrm{mg} \mathrm{kg}^{-1}$ and $0.1 \mathrm{mg} \mathrm{kg}^{-1}$. The existing analytical techniques

${ }^{a}$ Department of Food Sciences and Engineering, School of Chemical Engineering and Technology, Harbin Institute of Technology, 150090 Harbin, PR China. E-mail: zhaoht9999@163.com

${ }^{b}$ Key Laboratory of Agro-Product Quality and Safety, Institute of Quality Standard and Testing Technology for Agro-Product, Chinese Academy of Agricultural Sciences, 100081 Beijing, PR China

${ }^{c}$ National Research Center of Soybean Engineering and Technology, Northeast Agricultural University, Harbin 150030, PR China

$\dagger$ Electronic supplementary information (ESI) available. See DOI: 10.1039/c7ra05167k

\$ These authors contributed equally to this work and should be considered as co-first authors. for the analysis of thiamethoxam mainly include highperformance liquid chromatography (HPLC), ${ }^{6,7}$ liquid chromatography and mass spectrometry. ${ }^{8}$ Despite being sensitive and specific, all these methods require expensive equipment, highly trained personnel, and time-consuming pretreatment. So they can only be carried out in the laboratory and are not convenient for rapid screening on farmland and in the market. Moreover, these methods can involve high levels of material consumption, such as toxic organic solvents, which is again a serious environmental concern. For these reasons, electrochemical sensors that are highly sensitivity, have a fast response, are low cost, are easily portable and have other characteristics might represent an alternative solution. ${ }^{\mathbf{9}, 10}$

The recognition element on the surface of the electrode is the core of an effective electrochemical sensor. ${ }^{11}$ Due to the poor electrocatalytic performance of a glassy carbon electrode, we need to modify it. Graphene $(\mathrm{GN})^{\mathbf{1 0 , 1 2 - 1 4}}$ with high electrocatalytic activity is preferred, but graphene alone can't meet the need for detecting trace levels and specificity in pesticide analysis. Therefore, it is necessary to synergize with molecular imprinting technology to enhance its use. The molecular imprinting technique involves a kind of cross-linked polymer that exhibits specific binding sites around a template molecule to form a chemically and sterically complementary complex ${ }^{\mathbf{1 5}}$. Molecularly imprinted electrochemical sensors combine the superiorities of both molecularly imprinted polymers (MIPs) and electrochemical sensors, showing high sensitivity and selectivity, ease of preparation, simplicity, reusability, and the 
possibility of miniaturization and automation at low cost. ${ }^{\mathbf{1 6 , 1 7}}$ Based on the above, we use graphene as a carrier to prepare a graphene-based surface molecularly imprinted polymer as a recognition element, so as to improve the sensitivity and specificity of pesticide residue analysis.

Up to now, only a few papers have reported surface MIPs based on GN that have been constructed. The inert surface and poor dispersibility make it difficult to merge MIPs and $\mathrm{GN}^{\mathbf{1 8}}$. Zeng et al. developed a strategy for grafting molecularly imprinted films onto the graphene surface using the olefinic bonds of graphene itself. The electrochemical detection limit for 4-nitrophenol was $0.005 \mu \mathrm{M} .{ }^{19}$ However, graphene oxide has fewer functional groups, such as alkenyl groups, after reduction, so the molecular imprinting sites for grafting are limited. Luo et al. modified graphene with $N$-vinylcarbazole to make a surface rich in alkenyl groups, which improved selectivity significantly. ${ }^{20}$ Compared with Zeng's work, due to the addition of alkenyl modification reagents, the molecular imprinted film was thicker, reaching about $17 \mathrm{~nm}$. The molecular imprinting film is too thick to enhance the electrochemical signal. Luo et al. also developed a strategy to prepare graphene oxide (GO) based molecularly imprinted polymers (MIPs) for a glycoprotein electrochemical sensor, using boronic acid functionalized graphene oxide (GO-APBA) as a supporter by immobilizing them onto the surface of GO-APBA through boronate affinity. ${ }^{21}$ Chen et al. reported the novel design of magnetic two-dimensional molecularly imprinted polymers for the good recognition and separation of proteins, where dopamine was a reducing agent for graphene oxide. ${ }^{22}$

In summary, the study of surface molecularly imprinted electrochemical sensors with graphene as the carrier is in the initial stages. At present, graphene should be firstly pretreated, such as vinyl group functionalized graphene, to serve as a support, but more reagents and processes have been introduced. ${ }^{23,24}$ Luo et al. developed a novel graphene-molecularly imprinted polymer composite for bovine hemoglobin by simply mixing dopamine, graphene oxide and bovine haemoglobin (BHb) in aqueous solution; a thin adherent polydopamine film imprinted with $\mathrm{BHb}$ was spontaneously obtained on the surface of the graphene sheets, which was really convenient and easy to scale up. ${ }^{25}$ So the development of new graphenemodified reagents to study grafted molecular imprinting membranes has great theoretical value.

In this study, we present a facile method for the preparation of graphene based molecularly imprinted polymers (MIPs-GN) for the electrochemical detection of thiamethoxam (TMX) residue. The innovation of this study is in choosing $p$-vinylbenzoic acid as the functional monomer; the recognition units and alkenyl units can be added onto the surface of graphene via $\pi-\pi$ interactions in just one step, effectively simplifying the reaction process. And an ultra-thin imprinting film is formed by reducing the addition step involving the alkenyl modifier. Thanks to the irreversible electrochemical reduction of TMX, the template could easily leach out of the binding sites, and it does not require any organic reagents, only aqueous solution. Besides, the adsorption mechanism and the properties of the MIP layer on the GN surface are carefully discussed.
Furthermore, MIP-GN/GCE demonstrated efficiency in the detection of TMX in brown rice.

\section{Experimental}

\subsection{Reagents and apparatus}

Thiamethoxam (TMX) standard was obtained from YANGCHENG FLY HIGH CHEMICAL CO., LTD (China). Graphite powder, hydrazine hydrate, and 4-vinylbenzoic acid (VBA, 97\%) were purchased from Shanghai Aladdin Chemical Reagent Company (China). Ethylene glycol dimethacrylate (EGDMA, 98\%) was obtained from Sigma (China). 2,2-azobisisobutyronitrile (AIBN, 98\%) and $N, N$-dimethylformamide (DMF) were purchased from Kemiou Chemical Reagent Co (Tianjing, China). All other chemicals were of analytical grade and used as received without further purification. Doubly distilled water was used throughout the work.

Scanning electron microscopy (SEM) images were obtained on a Quantum 200FEG (FEI, U.S.). An Agilent Technology 1100 high performance liquid chromatograph (HPLC) connected to a diode array detector was employed. In addition, an Agilent- $\mathrm{C}^{\mathbf{1 8}}$ column $(4.6 \times 250 \mathrm{~mm}$, i.e., $5.0 \mathrm{~m})$ was used. All electrochemical measurements such as cyclic voltammetry (CV) and linear sweep voltammetry (LSV) measurements were performed using CHI 660E and GHI 1040C electrochemical work stations (Chenhua Instrument Co, Ltd, Shanghai, China), with threeelectrode systems. A conventional three-electrode system was composed of a MIP-GN or NIP-GN modified bare glassy carbon electrode (GCE, Ø $3 \mathrm{~mm}$ ) as the working electrode, an $\mathrm{Ag} / \mathrm{AgCl}$ ( $3 \mathrm{M} \mathrm{KCl}$ ) electrode as the reference electrode and a platinum wire as the counter electrode.

\subsection{Preparation of MIP-GN/GCE and NIP-GN/GCE}

Graphene (GN) was synthesized using a modified method reported by Park et al. ${ }^{26}$ After determining the preparation scheme for MIP-GN/GCE, the parameters of the molecularly imprinted polymerization system were optimized. We optimized the important factors, including the ratio of functional monomer (VBA) to template molecule (TMX), the ratio of functional monomer to carrier GN, the ratio of functional monomer to crosslinking agent (EGDMA), and the amount of initiator (AIBN) in the molecular imprinting system, determining the best parameters (as shown in Fig. S1-S4 $\dagger$ ). The experimental parameters and procedures are as follows: firstly, GN (10 mg) was scattered in $10 \mathrm{~mL}$ of $0.1 \mathrm{mmol} \mathrm{VBA} / \mathrm{DMF}$ solution and shake blended for $2 \mathrm{~h}$ at room temperature $\left(25^{\circ} \mathrm{C}\right)$ to prepare monomer (VBA) modified GN. Then, $10 \mathrm{~mL}$ of $0.1 \mathrm{mmol}$ TMX/DMF was added and mixed fully under shaking for $2 \mathrm{~h}$. Then, the cross-linker (EGDMA, $0.75 \mathrm{mmol}$ ) and initiator (AIBN, $0.025 \mathrm{~g}$ ) were added to a $20 \mathrm{~mL}$ dispersion of the functional monomer and template molecule, and this was stirred under nitrogen for $10 \mathrm{~min}$. The polymerization reaction was carried out at $60{ }^{\circ} \mathrm{C}$ for $24 \mathrm{~h}$. For comparison, blank non-molecularly imprinted composites (NIP-GN) were prepared under the same conditions only without the addition of a template molecule in the polymerization process. 
Prior to modification, the glassy carbon electrode (GCE) was polished successively in $0.5 \mu \mathrm{M}$ alumina slurries, rinsed thoroughly with doubly distilled water and dried in air. Then, the above synthesised MIP-GN $(5 \mu \mathrm{L})$ was dropped on the wellpolished GCE. Then it was dried at room temperature, dropped with $5 \mu \mathrm{L}$ of $0.1 \%$ chitosan (CS, dissolved in HAc) solution and dried again. Here MIP-GN/GCE was successfully prepared.

MIP-GN/GCE needs to have the template molecule removed. Firstly, in order to discard redundant reagents, MIP-GN/GCE was washed, under magnetic stirring, carefully with ethanol for $30 \mathrm{~s}$. Then, the template molecules of TMX were removed via CV scanning; MIP-GN/GCE was soaked in $0.1 \mathrm{M}$ phosphate buffered solution (PBS, pH 7.2) under CV over a potential scan range of -0.2 to $-1.4 \mathrm{~V}$ and with a scan rate of $0.05 \mathrm{~V} \mathrm{~s}^{-1}$ until there was no signal from TMX, and then it was rinsed thoroughly with DDW. This MIP-GN/GCE was used for electrochemical experiments. For comparison, NIP-GN/GCE was prepared under the same conditions.

\subsection{Electrochemical measurements}

For electrochemical measurements including cyclic voltammetry (CV) and linear sweep voltammetry (LSV), the three-electrode system was immersed in a $10 \mathrm{~mL}$ electrochemical cell containing $0.1 \mathrm{M}$ PBS solution and an appropriate concentration of TMX. After the extraction of the templates, MIP-GN/GCE was incubated in 0.1 M PBS containing different concentrations of TMX for 10 min and examined using LSV in new PBS. The linear sweep voltammograms were recorded from $-0.2 \mathrm{~V}$ to $-1.2 \mathrm{~V}$ and at a scan rate of $0.05 \mathrm{~V} \mathrm{~s}^{-1}$. The frequency range was $0.1 \mathrm{~Hz}$ to $10^{5} \mathrm{~Hz}$ for electrochemical impedance spectroscopy, which was conducted in $5 \mathrm{mM} \mathrm{Fe}(\mathrm{CN}) 6^{3-/ 4-}$ and $0.1 \mathrm{M} \mathrm{KCl}$ solution at a formal potential of $0.25 \mathrm{~V}$, with a signal amplitude of $5 \mathrm{mV}$.

\subsection{Sample preparation}

The organic brown rice sample was purchased from the local market and crushed, and was detected to contain no TMX. Three levels of standard TMX concentrations $(5.0 \mu \mathrm{M}, 10.0 \mu \mathrm{M}$, and $15.0 \mu \mathrm{M}$ ) were spiked into $1 \mathrm{~g}$ of sample, which was prepared using the efficient method established by our previous group. ${ }^{27}$

\section{Results}

\subsection{Preparation of MIP-GN/GCE}

The preparation of a glassy carbon electrode modified with molecularly imprinted polymers which are based on graphene (MIP-GN/GCE) is the core of this experiment, and is carried out mainly through three steps. Firstly, a TMX molecularly imprinted polymeric material based on graphene (MIP-GN) was prepared. Subsequently, a facile sensor was obtained by directly dropping the synthetic MIP-GN dispersion on a well-polished electrode. Finally, electrochemical cyclic voltammetry (CV) was used to remove the template molecules.

3.1.1. Protocol for MIP-GN/GCE. Fig. 1 presents an illustration of the easy preparation procedure for MIP-GN/GCE through the surface imprinting route. Firstly, graphene was used as a carrier. As is known, the introduction of double bonds onto the surface of graphene is cumbersome, complicated and time consuming during the course of molecular imprint preparation. We selected 4-vinylbenzoic acid (VBA) as a functional monomer, it can result in an ultra-thin imprinting film being formed by reducing the addition step involving the alkenyl modifier, making the preparation procedure much easier. On the one hand, VBA can be immobilized onto the surface of GN via $\pi-\pi$ interactions, and, furthermore, VBA itself contains both alkenyl and hydroxyl groups, so that it can connect with the template molecule and cross-linking agent. Thus, the reaction process was easier to control, and it was beneficial in reducing differences among product batches. Luo's group proposed vinyl-functionalized graphene modified with vinylcarbazole through $\pi-\pi$ interactions. ${ }^{24}$ Compared with their work, the greatest advantage of our work is that the recognition units (hydroxyl groups for connecting with template molecules) and alkenyl units (for connecting with the cross-linking agent) were modified onto the surface of GN in just one step. Furthermore, this is advantageous in forming a more uniform molecular imprinting layer on the surface of the graphene sheet, so that the sensor response signal is more stable. Then the template TMX was added to form the pre-polymerized product. Subsequently, molecularly imprinted polymers which were based on graphene (MIP-GN) were successfully obtained through copolymerization in the presence of a cross linker and initiator. After polymerization for $24 \mathrm{~h}$ at $60^{\circ} \mathrm{C}$, a facile sensor was obtained by directly dropping the resulting dispersion on a well-polished electrode. Chitosan solution was further dropped onto the molecularly imprinted layers to form a protective film, to prevent the MIP layer from falling off the glassy carbon electrode. Then, unreacted material was washed with ethanol and the template molecules were eluted in blank PBS solution via electrochemical methods. A glassy carbon electrode modified with non-molecularly imprinted polymers based on graphene (NIP-GN/GCE) was prepared using the same procedure, just without the procedure of template molecule (thiamethoxam) addition.

3.1.2. Template removal in MIP-GN/GCE using CV. Suitable removal of the template in MIP-GN/GCE contributes to producing a structurally-related template in the polymeric matrix. It is well known that conventional template elution using methanol/acetic acid mixed solutions has many shortcomings. It is time-consuming and requires abundant organic reagents, leading to higher detection costs, as well as not being environmentally friendly. ${ }^{15}$ Due to the irreversible electrochemical reduction properties of TMX, it can be eluted using electrochemical cyclic voltammetry. ${ }^{28}$ As shown in Fig. 2a and b, compared with NIP-GN/GCE, there is a typical peak at $-1.0 \mathrm{~V}$ to $-1.2 \mathrm{~V}$ for MIP-GN/GCE during the first scanning sweep, indicating that this peak is the characteristic peak of TMX. As shown in Fig. 2c, we can see that the pretty high peak current of TMX almost disappears in the second cycle of scanning. Following an increasing number of scans, the TMX peak response signal is not present, indicating that the template molecule has been completely eluted. Compared with traditional chemical elution methods, this method is not only fast, but it does not require 

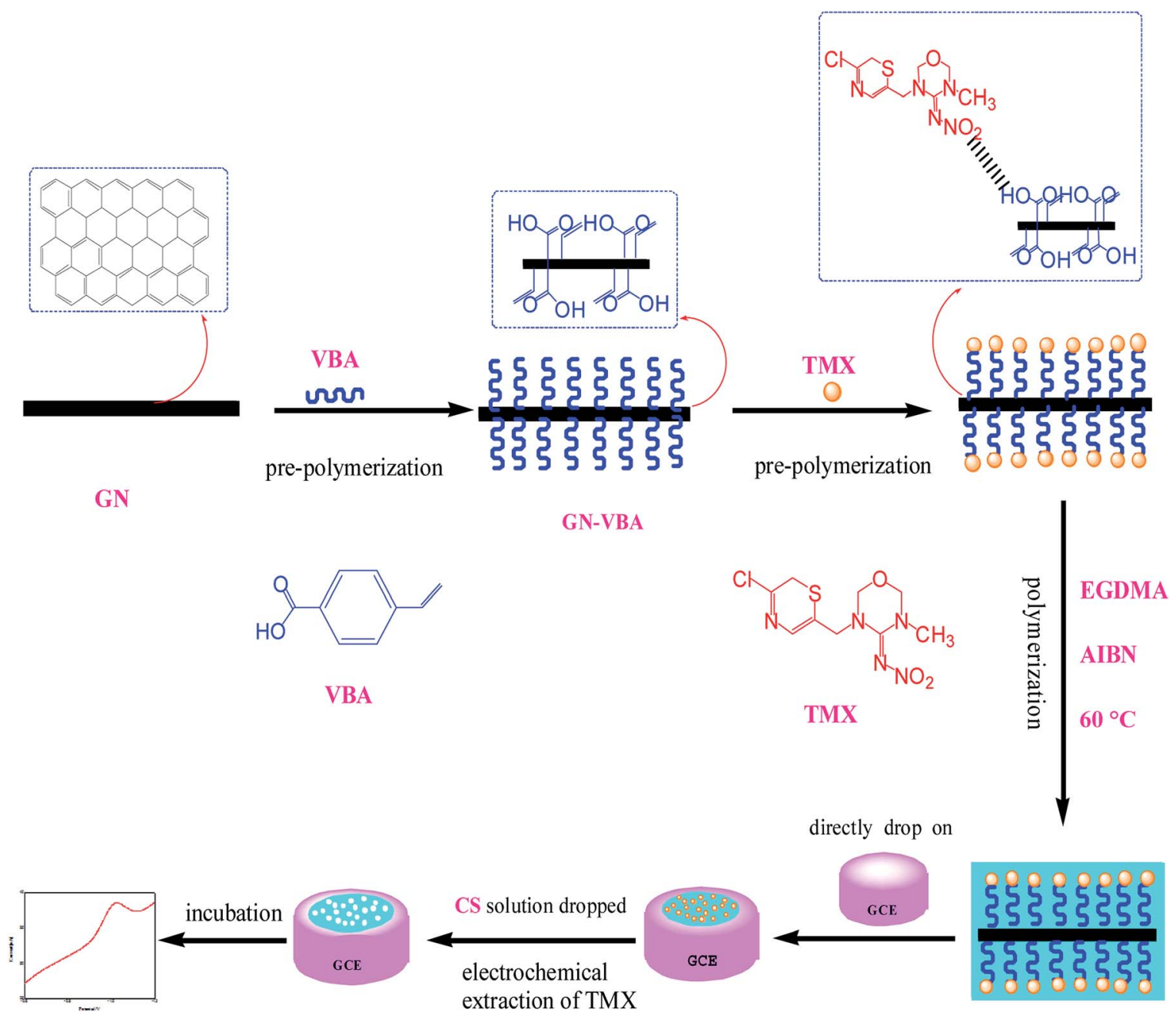

Fig. 1 Schematic diagram of the procedure for the preparation of MIP-GN/GCE and our concept for the selective electrochemical detection of TMX.

any organic reagents and can monitor in real time whether the template molecule has been completely eluted.

3.1.3. The protective effect of chitosan. In the process of electrochemical tests, we have the problem that the MIP-GN film was somewhat rigid, and may fall off the glassy carbon electrode, resulting in an unsteady electrochemical signal. So we included the dropping of a layer of protective film to prevent the imprinted film from falling off, while not blocking electron transfer. After consideration of its excellent film-forming abilities and porousness, CS solution was used to protect the MIPGN film. As shown in Fig. 3a, the effects of different CS concentrations on the response peak current from TMX using MIP-GN/GCE were studied. It can be seen that the peak current increases significantly with an increase in CS concentration from $0.05 \%$ to $0.1 \%$, and then decreases sharply with a continuous increase in CS concentration. The possible reason for this is that the chitosan can't effectively protect the imprinted film at low concentrations. In contrast, a high concentration of chitosan can hinder electron transport, resulting in a significant decrease in peak current values, so we use $0.1 \%$ CS to protect the MIP-GN/GCE film. In order to further determine whether
$0.1 \%$ CS has an inhibitory effect on electron transport, it is compared with the peak current from TMX on GN/GCE without CS film. As shown in Fig. 3b, there was no difference in the peak current between GN/GCE and CS/GN/GCE, demonstrating that there was no hindering of electron transfer, and effectively solving the problem of the film falling off the glassy carbon electrode.

\subsection{Characterization of MIP-GN/GCE}

The morphologies of GN/GCE and MIP-GN/GCE were investigated using scanning electron microscopy (Fig. 4). GN sheets showed typically curved, layered structures with fairly smooth surfaces. The typical curved structure can provide a high surface area, providing suitable support for the molecular imprinting process. Compared with GN/GCE, MIP-GN/GCE showed a dense and rather rough surface, indicating that MIP films were uniformly loaded on the GN surface and MIP films were successfully grafted onto the GN surface. The surfaces of the MIP films were further probed using AFM (Fig. 5). The height of the GN as measured from cross section analysis was in the order 

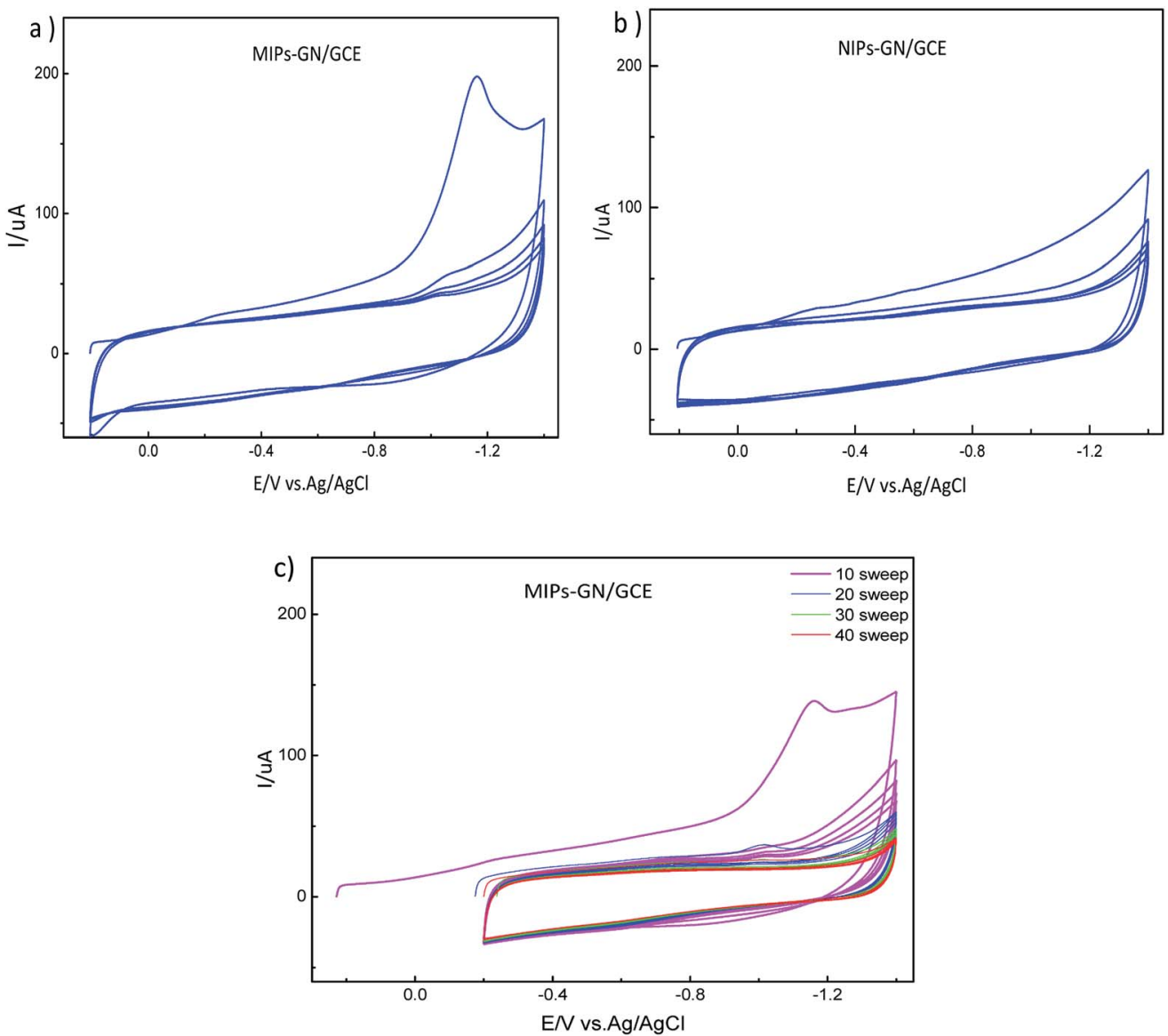

Fig. 2 Repetitive cyclic voltammograms of (a) MIP-GN/GCE; (b) NIP-GN/GCE; and (c) MIP-GN/GCE in blank PBS with different cycle numbers: $10,20,30$, and 40 .

of $0.790 \mathrm{~nm}$, and thus the image corresponds to single-layer nano-sheets. According to AFM imaging of MIP-GN, the average thickness of the polymer grafted on the GN surface is about $4.793 \mathrm{~nm}$, indicating that an imprinted film was successfully synthesized. Since both sides of the graphene sheet are loaded with molecularly imprinted films, the thickness of the molecularly imprinted film is about $2.0 \mathrm{~nm}$, which is much lower than the reported molecularly imprinted film thickness (17 nm) on a graphene surface in the literature. Because the addition step involving an alkenyl-modifying agent is omitted, an ultra-thin imprinted film is formed. An ultra-thin imprinted film is conducive to the transmission of electrons and improves detection sensitivity. Fig. 6 shows electrochemical impedance spectroscopy (EIS) data for MIP-GN/GCE removable template
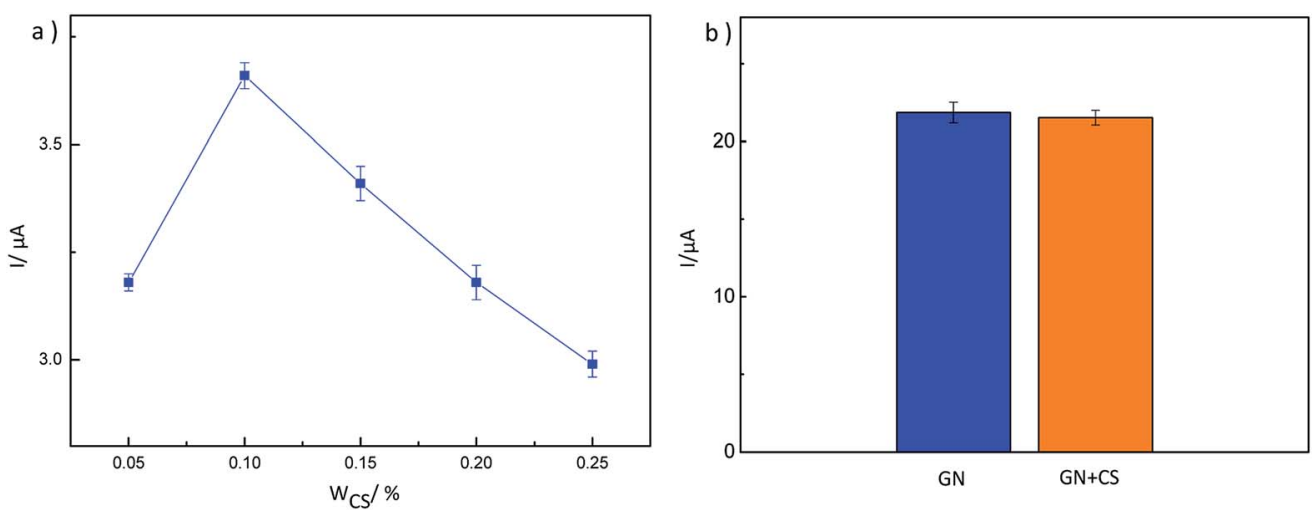

Fig. 3 (a) A plot of peak current vs. concentration of CS; and (b) LSV data for GN/GCE and CS (0.1\%) + GN/GCE after incubating for 10 min in $5 \mu M$ TMX, recorded in blank PBS. 

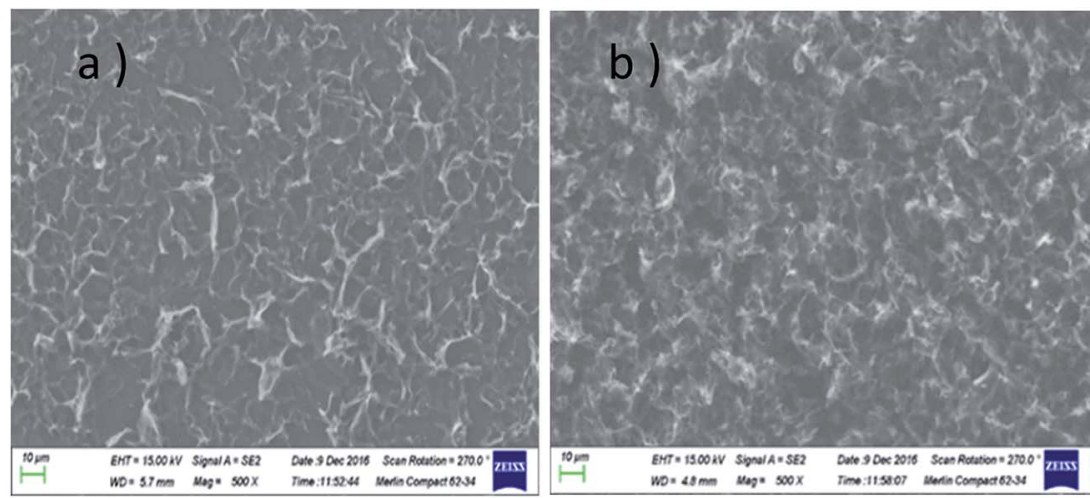

Fig. 4 SEM images of (a) GN/GCE, and (b) MIP-GN/GCE.

molecules, MIP-GN/GCE and NIP-GN/GCE in $5.0 \mu \mathrm{M}$ $[\mathrm{Fe}(\mathrm{CN}) 6]^{3-/ 4-}$ containing $0.1 \mathrm{M} \mathrm{KCl}$ solution. All EIS results include a semicircular region lying on the axis of the plot, followed by a straight line for the three electrodes. The semicircular region observed at higher frequencies corresponds to the electrode transfer-limited process, thus the semicircle diameter equals the surface electrode transfer resistance (Ret). Obviously, the Ret of MIP-GN/GCE becomes lower after the removal of the template molecules. This result was because when the templates were removed from the electrode surface, specific recognition sites appeared. The Ret of MIP-GN/GCE with removable template molecules is also less than NIP-GN/GCE, indicating the high electron transfer rate for MIP-GN films.

\subsection{Performance of MIP-GN/GCE}

The adsorption mechanism for MIP-GN/GCE and nicotine pesticides was studied via an adsorption isotherm study and an adsorption kinetic study. The selectivity, linear response, stability and reusability of MIP-GN/GCE were also evaluated, along with interference and repeatability studies.

3.3.1. Electrochemical behaviour of MIP-GN/GCE and NIPGN/GCE. After incubating in $5 \mu \mathrm{M}$ TMX solution for $10 \mathrm{~min}$ and washing in water under magnetic stirring, the electrochemical behaviour of MIP-GN/GCE and NIP-GN/GCE was investigated in blank PBS buffer ( $\mathrm{pH}$ 7.2) solutions using linear sweep voltammetry (LSV). As shown in Fig. 7, a distinct characteristic peak from TMX in the MIP-GN modified GCE data (curve a) was observed at around $-1.0 \mathrm{~V}$, which corresponds to the reduction of the nitro group in the structure of TMX to an amino group via four-electron transfer. In comparison, NIP-GN/GCE exhibits a relatively much smaller signal response at $-1.0 \mathrm{~V}$ (curve b), which should be attributed to non-specifically adsorbed TMX molecules on the NIP-GN film. The imprinting factor (IF = AMIP-GN/ANIP-GN) was 2.36, indicating that there were a large
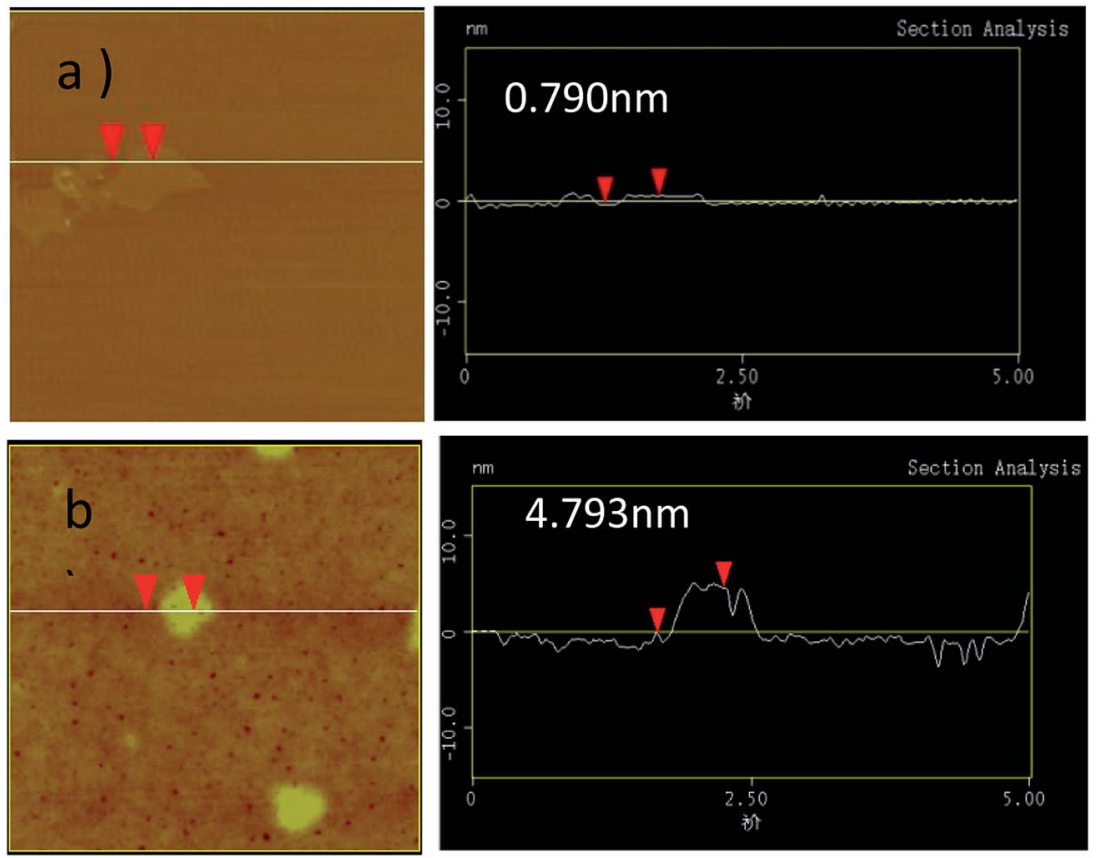

Fig. 5 AFM data for (a) GN, and (b) MIP-GN. 


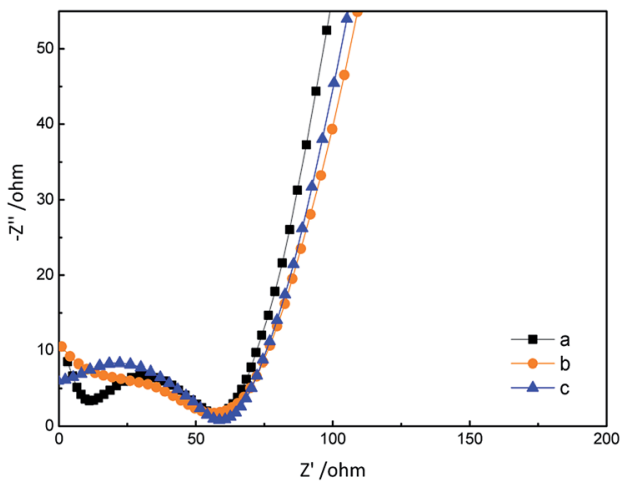

Fig. 6 Electrochemical impedance spectroscopy (EIS) data for (a) MIP-GN/GCE with removable template molecules; (b) MIP-GN/GCE; and (c) NIP-GN/GCE.

number of binding sites on the MIP-GN film, which would allow it to adsorb more target molecules. In addition, an unmodified GCE was also applied as a control group (curve c). The bare GCE presented a minimal electrochemical signal in the absence of highly electro-catalytic GN.

3.3.2. Adsorption isotherms. The effects of adsorbate concentration on the adsorption capacity were studied using adsorption thermodynamics. The thermodynamic adsorption of MIP-GN/GCE was studied over the concentration range 0.05$50 \mu \mathrm{M}$. Fig. 8 shows the adsorption isotherms for TMX on MIPGN/GCE and NIP-GN/GCE. It can be seen that the peak current from TMX increases with increasing concentration. At the same time, the peak current of MIP-GN/GCE is always greater than NIP-GN/GCE, indicating that MIP-GN/GCE has a higher specificity to TMX. In addition, we also find that when the concentration increased to $10-20 \mu \mathrm{M}$, the IF began to decrease, which is due to non-specific adsorption by NIP-GN/GCE. Besides, the LSV data presented as double-peaks when the concentration was above $60 \mu \mathrm{M}$ (Fig. S5 $\dagger$ ). It was speculated that the new peak at about $-1.1 \mathrm{~V}$ was due to physical adsorption, when special recognition absorption achieved saturation. In order to further understand the mechanism of adsorption, adsorption isotherm models, which describe the manner in which molecules interact with the surface of the modified GCE, were developed in this study. ${ }^{29}$ As shown in Fig. 9, the correlation coefficient of the Langmuir isotherm was 0.980 , and the correlation coefficient of the Freundlich model was 0.996 ; both models have good fitting. The Langmuir isotherm indicates that the adsorbent surface is covered by a monolayer of molecules in a horizontal position. It assumes that the adsorbent layer is structurally homogeneous and all the active sites are identical and energetically equivalent. The binding sites will not adsorb template molecules further once the template molecules occupy the sites. So, the high correlation of the Langmuir model reveals that the MIP layer on the GN surface is a monolayer. And the $1 / n$ value from the Freundlich model is less than 1, indicating that the MIP-GN adsorption process is preferred.

3.3.3. Adsorption kinetics. In order to investigate the adsorption rate and equilibrium of the molecularly imprinted polymers, adsorption kinetic curves for MIP-GN/GCE and

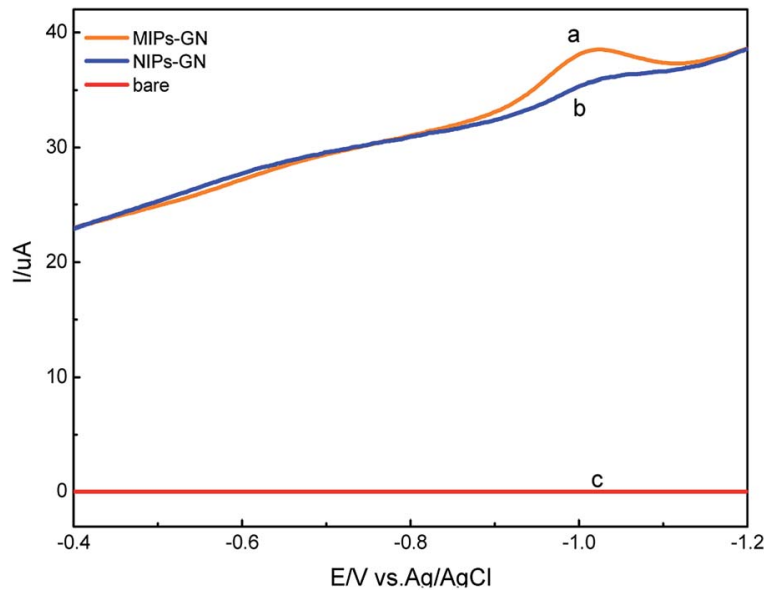

Fig. 7 LSV data for (a) MIP-GN/GCE, (b) NIP-GN/GCE, and (c) a bare glassy carbon electrode, recorded in PBS buffer (pH 7.2) after incubation in $5 \mu \mathrm{mol} \mathrm{L} \mathrm{L}^{-1} \mathrm{TMX}$ solution for $10 \mathrm{~min}$.

NIP-GN/GCE with $5 \mu \mathrm{M}$ TMX were recorded, as shown in Fig. 10. It can be seen that the peak current from MIP-GN/GCE increases rapidly in the first 10 minutes and then remains essentially unchanged, indicating that the binding of MIP-GN/GCE to TMX reached a dynamic equilibrium. At the same time, the response of NIP-GN/GCE to TMX is to grow slowly over time, resulting in the IF beginning to reduce after 12-15 min. The reason for this was that adsorption on NIP-GN/GCE is physical adsorption, and a longer incubation time leads to increasing non-specific adsorption. Unlike NIP-GN/GCE, the MIP layer on the GN surface is a monolayer structure. Abundant TMX molecules coated on the modified electrode would not be recognized by the MIP layer anymore, resulting in a lower IF value. The comparison shows that the binding of MIP-GN/GCE to TMX can be quickly balanced. Hence, the adsorption was specific and binding equilibrium was achieved after an incubation time of about $10 \mathrm{~min}$, indicating quite fast binding processes.

3.3.4. Selectivity of MIP-GN/GCE. Selectivity experiments were carried out with interfering species which always accompany TMX in real samples and have similar structures. We

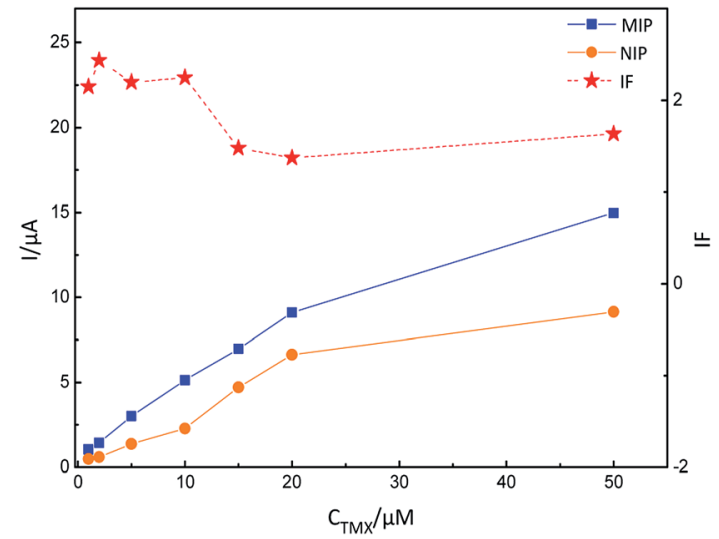

Fig. 8 Adsorption dynamics curves for TMX on MIP-GN/GCE and NIP-GN/GCE. 

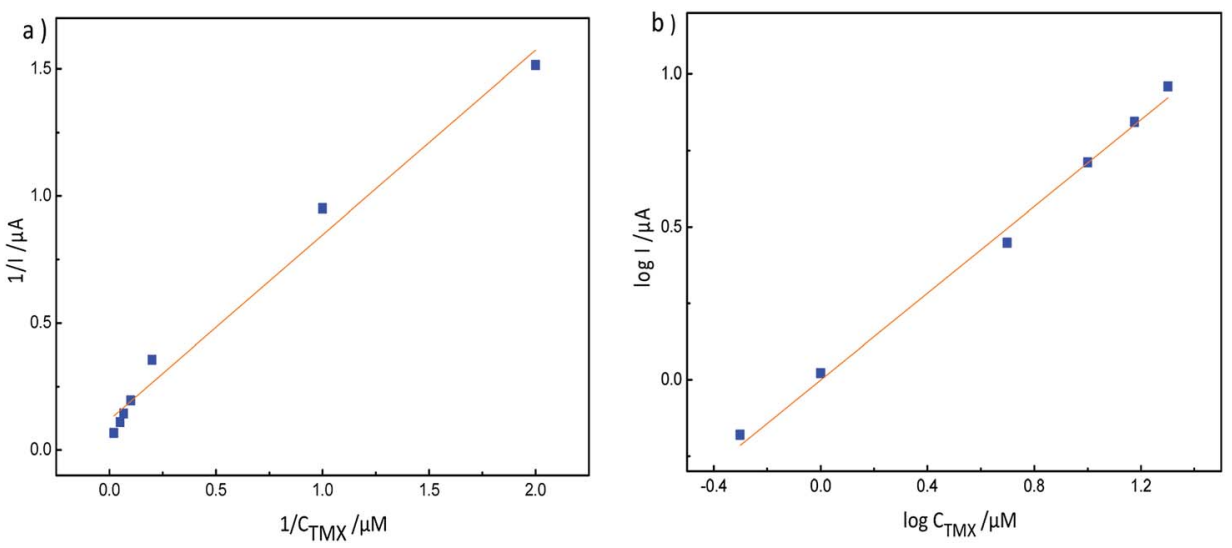

Fig. 9 (a) The Langmuir model for MIP-GN/GCE adsorption; and (b) the Freundlich model for MIP-GN/GCE adsorption.

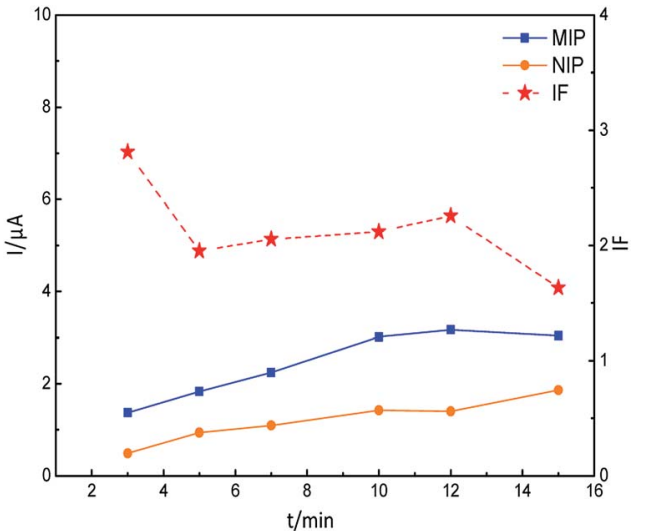

Fig. 10 Adsorption dynamics curves for TMX on MIP-GN/GCE and NIP-GN/GCE.

considered the same class of neonicotinoid pesticides, which have the most similar structures, including acetamiprid, nitenpyram, dinotefuran, and thiacloprid. Among these four, acetamiprid and thiacloprid have no influence on TMX determination because the absence of a nitro group makes them have no electrochemical activity. Besides, we also have chosen other electrochemically active pesticides like parathion-methyl and Dursban. However, these pesticides have different sensing signal strengths owing to the discrepancy of their structures. In order to realize the current response discrepancy from TMX and its analogs, LSV curves of TMX and its analogs on GN/GCE after incubation were recorded in PCM-free solutions. As shown in Fig. 11a, a distinct characteristic peak from TMX at around $-1.0 \mathrm{~V}$ was observed. Other pesticides, including parathion-methyl, nitenpyram and dursban, have no influence on practical analysis, as their reduction peak potentials are at around $-0.6 \mathrm{~V}$ and their sensing signals were much less than TMX. However, the response signal at around $-1.1 \mathrm{~V}$ from dinotefuran was close to TMX. So we researched the selective current response of TMX, dinotefuran, parathion-methyl, nitenpyram and dursban on MIP-GN/GCE and NIP-GN/GCE. The LSV current response of TMX on MIP-GN/GCE was nearly 2.5 times higher than on NIP-GN/GCE (Fig. 11b). Similarly, the currents of the other 3 analogs on MIP-GN/GCE were higher than on NIP-GN/GCE. But the IFs were 1.33, 1.07, and 0.95, respectively, indicating that MIP-GN/GCE had good specific recognition abilities toward TMX.
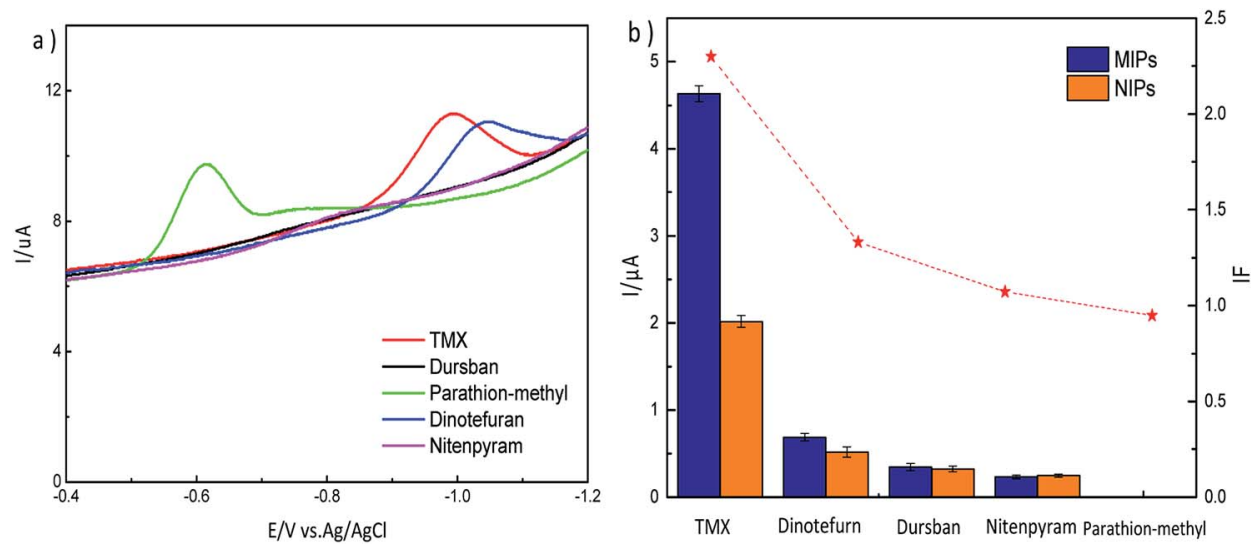

Fig. 11 (a) LSV curves for TMX and its analogs on GN/GCE after incubation for 10 minutes; and (b) the selective LSV current response for $5 \mu M$ TMX and its analogs on MIP-GN/GCE and NIP-GN/GCE after incubation for 10 minutes. 

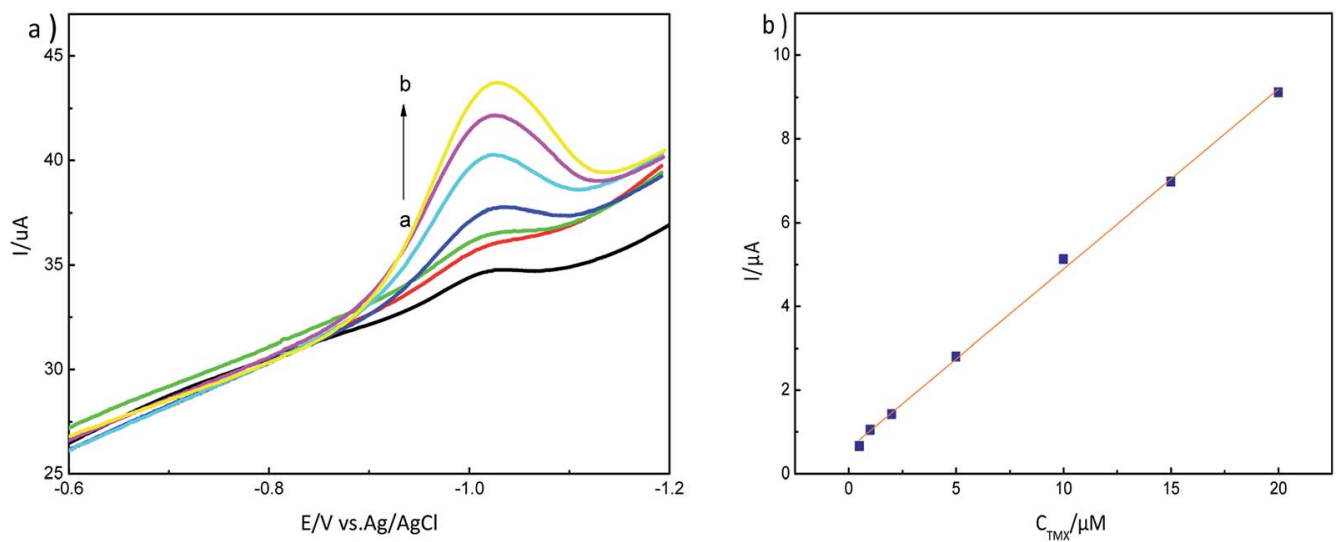

Fig. 12 (a) LSV response to different concentrations of thiamethoxam, 0.5-20 $\mu \mathrm{M}(\mathrm{a} \rightarrow \mathrm{b})$, on an MIP-GN modified electrode in PBS buffer (pH 7.2); and (b) the calibration curve for TMX on MIP-GN/GCE.

Table 1 Comparison of the sensing performance for thiamethoxam of various electrodes

\begin{tabular}{|c|c|c|c|c|}
\hline Electrochemical method & Electrode & Linear range & LOD & Reference \\
\hline Cyclic voltammetry & GCE & $95-1714 \mu \mathrm{M}$ & $29 \mu \mathrm{M}$ & Guzsvány et $a l^{28}$ \\
\hline Differential pulse polarography & Bismuth film electrode (GCE) & $4.3-154.3 \mu \mathrm{M}$ & $1.3 \mu \mathrm{M}$ & Guzsvány et al. ${ }^{32}$ \\
\hline Differential pulse polarography & Dropping mercury electrode (DME) & $0.11-1.61 \mu \mathrm{M}$ & $0.032 \mu \mathrm{M}$ & Guzsvány et $a l .{ }^{33}$ \\
\hline Differential pulse polarography & Nanosilver/SDS/GCE & $0.1-9 \mu \mathrm{M}$ & $0.1 \mu \mathrm{M}$ & Kumaravel et al. ${ }^{34}$ \\
\hline Cyclic voltammetry & Nanosilver/SDS/GCE & $10-90 \mu \mathrm{M}$ & $4.7 \mu \mathrm{M}$ & Kumaravel et al. ${ }^{34}$ \\
\hline
\end{tabular}

3.3.5. LSV response and analytical curve. Typical linear sweep voltammetry responses for various concentrations of thiamethoxam are shown in Fig. 12a. The peak current increases with an increase in thiamethoxam concentration. We did a statistical analysis of thiamethoxam concentration over the range from $0.5 \mu \mathrm{M}$ to $20 \mu \mathrm{M}$ based on International Union Of Pure And Applied Chemistry (IUPAC) standards (using IBM SPSS Statistics software $)^{30}$. The results of the analysis show the

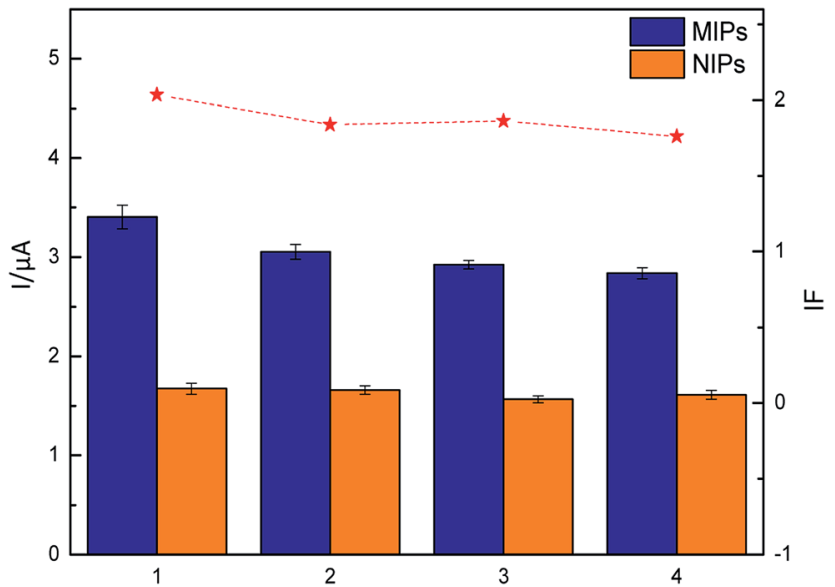

Fig. 13 LSV responses for a single MIP-GN/GCE electrode, repeated four times, which was incubated in $5 \mu \mathrm{MTMX}$. expected results; $F=3378.450$ and Sig. $=0.000$. This indicates that the regression relationship is statistically significant, the regression equation is generally significant, and the regression equation is effective, with $R=0.9991$. It is shown that the regression line has good goodness of fit with the sample observations. Thus, we obtained the regression equation $I=$ $0.430 C+0.596, R=0.9991$. As shown in Fig. $12 \mathrm{~b}$, it can be also observed that the peak current varies linearly with an increase in the concentration of thiamethoxam over the range from 0.5 $\mu \mathrm{M}$ to $20 \mu \mathrm{M}$. The limit of detection (LOD) has been determined

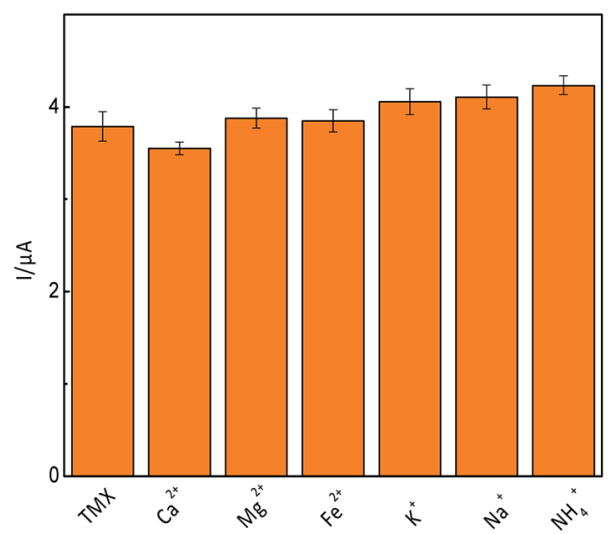

Fig. 14 Influence of various inorganic ions on the reduction of TMX $(5 \mu M)$. 
Table 2 Recovery of thiamethoxam at three spiked levels from brown rice

\begin{tabular}{llllll}
\hline & $\begin{array}{l}\text { Amount added } \\
\left(\mu \mathrm{mol} \mathrm{L} \mathrm{L}^{-1}\right)\end{array}$ & $\begin{array}{l}\text { Amount detected using } \\
\text { HPLC }\left(\mu \mathrm{mol} \mathrm{L} \mathrm{L}^{-1}\right) \pm \mathrm{SD}\end{array}$ & $\begin{array}{l}\text { Recovery rate using } \\
\text { HPLC }(\%) \pm \mathrm{SD}\end{array}$ & $\begin{array}{l}\text { Amount etected using } \\
\text { LSV }\left(\mu \mathrm{mol} \mathrm{L}^{-1}\right) \pm \mathrm{SD}\end{array}$ & $\begin{array}{l}\text { Recovery rate using } \\
\text { LSV }(\%) \pm \mathrm{SD}\end{array}$ \\
\hline \multirow{2}{*}{ Brown Rice } & 5 & $4.77 \pm 0.09$ & $95.38 \pm 1.75$ & $4.49 \pm 0.4$ & $89.67 \pm 8.03$ \\
& 10 & $9.56 \pm 0.15$ & $95.62 \pm 1.53$ & $9.41 \pm 0.28$ & $93.99 \pm 2.74$ \\
& 15 & $14.16 \pm 0.26$ & $94.76 \pm 1.19$ & $13.31 \pm 0.04$ & $88.69 \pm 0.25$
\end{tabular}

to be $0.040 \mu \mathrm{M}$, according to modern standards accepted by IUPAC. ${ }^{31}$ The LOD of MIP-GN/GCE is lower when compared with other reported electrochemical methods for thiamethoxam detection (Table 1). The detection limit is comparable with a mercury based electrode reported in the literature. However, mercury is toxic and it is not environmentally friendly.

3.3.6. Reusability and repeatability of MIP-GN/GCE. The important reusability features of MIP-GN are desorption and regeneration. In this study, the adsorption and desorption cycle was repeated four times using a single MIP-GN/GCE electrode. As shown in Fig. 13, MIP-GN/GCE lost only $10-17 \%$ of its response signal on average over four cycles. A report on a magnetic-graphene based molecularly imprinted polymer nanocomposite for the recognition of bovine hemoglobin showed less than a $13.7 \%$ decrease after three cycles of adsorption-desorption. ${ }^{35}$ For the water-compatible surface imprinting of 'saccharin sodium' on a silica surface for selective recognition and detection in aqueous solution, it has been reported that the saccharin sodium-MIP lost $12-15 \%$ of its adsorption capacity on average over five cycles. ${ }^{36}$ The reusability of our MIP-GN/GCE is compared with other reported methods using MIPs, demonstrating high stability and maintaining almost the same adsorption capacity for the template TMX. In addition, the repeatability of MIP-GN/GCE was determined through replicate testing of the response values for different batch electrodes to the same sample ( $5 \mu \mathrm{M}$ TMX). Good repeatability was found, with an RSD value of $3.08 \%$ for 3 successive determinations, with the peak current from MIP-GN/GCEs being $4.636 \mu \mathrm{A}, 4.814 \mu \mathrm{A}$ and $4.530 \mu \mathrm{A}$.

3.3.7. Interference studies. The presence of interfering species, which can reduce the electrochemical sensing signal, is a major challenge for carrying out real sample analysis. The possible interference of several inorganic species on the reduction signal from $5 \mu \mathrm{M}$ TMX, with or without $5 \mu \mathrm{M}$ concentrations of inorganic ions $\left(\mathrm{Ca}^{2+}, \mathrm{Mg}^{2+}, \mathrm{Fe}^{2+}, \mathrm{K}^{+}, \mathrm{Na}^{+}\right.$and $\mathrm{NH}^{4+}$ ), was investigated using linear sweep voltammetry. The results (Fig. 14) showed that the presence of inorganic ion groups didn't create a significant difference to the control group, indicating that MIP-GN/GCE has strong anti-interference abilities.

\subsection{Application to real sample analysis}

To evaluate the practical performance of MIP-GN/GCE for the determination of TMX, rice samples were used. The recoveries obtained using electroanalytical techniques are shown in Table 2. There was no significant difference between the proposed sensor and HPLC analysis methods. A satisfactory result was also achieved with a minimum added concentration of $5 \mu \mathrm{mol} \mathrm{L}{ }^{-1}$ (equal to $0.001 \mathrm{mg} \mathrm{kg}^{-1}$ of sample weight). The results indicate the practicability of our MIP-GN/GCE sensor, which can be used for the determination of thiamethoxam residue in brown rice samples.

\section{Conclusions}

In conclusion, we present a facile method for the preparation of ultra-thin molecularly imprinted polymers based on graphene for the sensitive and selective determination of TMX residue. We selected 4-vinylbenzoic acid (VBA) as a functional monomer, so that the recognition units and alkenyl units were added onto the surface of GN in just one step. It was essential to simplify the reaction process on the electrochemical sensor using functional monomer modification. The ultra-thin imprinting film $(2 \mathrm{~nm})$ was formed by reducing the addition step involving the alkenyl modifier. The ultra-thin imprinted film is conducive to the transmission of electrons and improves detection sensitivity. The experimental results showed that MIP-GN/GCE not only possessed good sensitivity and binding capacity for TMX, but it also exhibited good reproducibility and reusability. In addition, the novel method was further applied to the detection of TMX in brown rice samples with satisfactory results.

\section{Acknowledgements}

This work was supported by the National Natural Science Foundation of China (No. 31571798, No. 31401618, and No. 31000831), the Natural Science Foundation of Heilongjiang Province (C2016033), the National Science Foundation for Postdoctoral Scientists of China (2014M551260), the Natural Science Research and Innovation Funds of Harbin Institute of Technology (HIT.NSRIF.2014093), Harbin Young talent reserve project 2014RFYJ179 and the Youth Science Fund Project of Heilongjiang Province (QC2013C026).

\section{Notes and references}

1 P. Jeschke and R. Nauen, Pest Manage. Sci., 2008, 64, 10841098.

2 A. Kumaravel and M. Chandrasekaran, Sens. Actuators, B, 2012, 174, 380-388.

3 R. Nauen, P. Jeschke and L. Copping, Pest Manage. Sci., 2008, 64, 1081.

4 D. Goulson and D. Kleijn, J. Appl. Ecol., 2013, 50, 977-987.

5 J. Kimurakuroda, Y. Komuta, Y. Kuroda, M. Hayashi and H. Kawano, PLoS One, 2012, 7, e32432. 
6 P. Wang, X. Yang, J. Wang, J. Cui, A. J. Dong, H. T. Zhao, L. W. Zhang, Z. Y. Wang, R. B. Xu, W. J. Li, Y. C. Zhang, H. Zhang and J. Jing, Food Chem., 2012, 134, 1691-1698.

7 S. Seccia, P. Fidente, D. Montesano and P. Morrica, J. Chromatogr. A, 2008, 1214, 115-120.

8 M. A. Di, P. Fidente, D. A. Barbini, R. Dommarco, S. Seccia and P. Morrica, J. Chromatogr. A, 2006, 1108, 1-6.

9 C. Malitesta, E. Mazzotta, R. A. Picca, A. Poma, I. Chianella and S. A. Piletsky, Anal. Bioanal. Chem., 2011, 402, 18271846.

10 J. Wackerlig and P. A. Lieberzeit, Sens. Actuators, B, 2015, 207, 144-157.

11 L. Uzun and A. P. Turner, Biosens. Bioelectron., 2016, 76, 131144.

12 Y. Zeng, Y. Zhou, L. Kong, T. Zhou and G. Shi, Biosens. Bioelectron., 2013, 45, 25-33.

13 Y. Zeng, Y. Zhou, T. Zhou and G. Shi, Electrochim. Acta, 2014, 130, 504-511.

14 F. Duan, C. Chen, G. Wang, Y. Yang, X. Liu and Y. Qin, RSC Adv., 2014, 4, 1469-1475.

15 L. Chen, X. Wang, W. Lu, X. Wu and J. Li, Chem. Soc. Rev., 2016, 45, 2137.

16 Y. Yang, Y. Cao, X. Wang, G. Fang and S. Wang, Biosens. Bioelectron., 2015, 64, 247-254.

17 M. L. Yola, T. Eren and N. Atar, Sens. Actuators, B, 2015, 210, 149-157.

18 T. Kuila, S. Bose, P. Khanra, A. K. Mishra, N. H. Kim and J. H. Lee, Biosens. Bioelectron., 2011, 26, 4637-4648.

19 Y. Zeng, Y. Zhou, L. Kong, T. Zhou and G. Shi, Biosens. Bioelectron., 2014, 130, 504-511.

20 J. Luo, J. Cong, J. Liu, Y. Gao and X. Liu, Anal. Chim. Acta, 2015, 864, 74-84.

21 J. Luo, J. Huang, J. Cong, W. Wei and X. Liu, ACS Appl. Mater. Interfaces, 2017, 9(8), 7735-7744.
22 F. Chen, W. Zhao, J. Zhang and J. Kong, Phys. Chem. Chem. Phys., 2015, 18, 718-725.

23 Y. Mao, Y. Bao, S. Gan, F. Li and L. Niu, Biosens. Bioelectron., 2011, 28, 291-297.

24 J. Luo, J. Cong, J. Liu, Y. Gao and X. Liu, Anal. Chim. Acta, 2015, 864, 74-84.

25 J. Luo, S. Jiang and X. Liu, Sens. Actuators, B, 2014, 203, 782789.

26 J. A. Sungjin Park, I. Jung, R. D. Piner, S. J. An, X. Li, A. Velamakanni and R. S. Ruoff, Nano Lett., 2009, 9, 15931597.

27 M. Zhang, H. T. Zhao, X. Yang, A. J. Dong, H. Zhang, J. Wang, G. Y. Liu and X. C. Zhai, Sens. Actuators, B, 2016, 229, 190199.

28 V. J. Guzsvány, F. F. Gaál, L. J. Bjelica and S. N. Ökrész, J. Serb. Chem. Soc., 2005, 70, 735-743.

29 M. Zhang, H. T. Zhao, X. Yang, W. T. Zhang, J. Wang, G. Y. Liu, H. Zhang and A. J. Dong, RSC Adv., 2016, 6, 3714-3722.

30 O. F. Pure and A. Chemistry, Pure Appl. Chem., 1998, 9931014.

31 L. A. Currie, Pure Appl. Chem., 1995, 1699-1723.

32 V. Guzsvány, M. Kádár, F. Gaál, L. Bjelica and K. Tóth, Electroanalysis, 2010, 18, 1363-1371.

33 V. Guzsvány, M. Kádár, F. Gaál, K. Tóth and L. Bjelica, Microchim. Acta, 2006, 154, 321-328.

34 A. Kumaravel and M. Chandrasekaran, Sens. Actuators, B, 2012, 174, 380-388.

35 J. Guo, Y. Wang, Y. Liu, C. Zhang and Y. Zhou, Talanta, 2015, 144, 411-419.

36 S. Zhao, X. Yang, H. Zhao, A. Dong, J. Wang, M. Zhang and W. Huang, Talanta, 2015, 144, 717-725. 